Journal of Computer Science 8 (4): 460-467, 2012

ISSN 1549-3636

(C) 2012 Science Publications

\title{
Cross-Layer Design for Reducing the Energy Consumption based on Mobility Parameter in DSR for Mobile Ad-Hoc Network
}

\author{
${ }^{1}$ Bhanumathi, V. and ${ }^{2}$ R. Dhanasekaran \\ ${ }^{1}$ Department of Electronics and Communication Engineering, \\ Anna University of Technology Coimbatore, Coimbatore, India \\ ${ }^{2}$ Department of EEE, Syed Ammal Engineering College, Ramanathapuram, India
}

\begin{abstract}
Problem statement: The main idea is to design an energy efficient algorithm for reducing the amount of overhearing based on RSS value in a Mobile Ad-hoc Network (MANET). Approach: A cross layer framework is designed by combining the physical, MAC and network layer. RSS value is taken from the physical layer and it is used for finding the Mobility Parameter (MP). MP value determines the level of overhearing and rebroadcast probability. The energy conservation is achieved by integrating the 802.11 PSM with DSR. In 802.11 PSM, all the nodes are in low power sleep state, but the disadvantage here is unconditional overhearing. So, a conditional overhearing mechanism is proposed based on mobility parameter to reduce the energy consumption. This mechanism reduces energy consumption but at the same time, the node mobility results in more stale routes in route cache of DSR over a period of time. This occurs due to the lack of route cache updation. In the proposed design, RSS value determines whether to select the link or not prior to the route discovery process. Results: Simulation results are compared for Packet Delivery Ratio (PDR) and energy consumption. MP based conditional overhearing shows a 3.2\% PDR improvement. It achieves a $20 \%$ power saving, if the pause time crosses $300 \mathrm{sec}$. For the packet injection rate of 2 packets/sec, under no mobility condition, it utilizes 50 joules lesser energy consumption compared to Random Cast. Conclusion: The NS-2 simulation results reveal that the proposed work is energy efficient by reducing the amount of overhearing and also it eliminates the stale routes compared to the classical methods.
\end{abstract}

Key words: MANET, DSR, stale route, RSS value, mobility parameter and overhearing

\section{INTRODUCTION}

In mobile ad-hoc network, the nodes are mobile and can communicate dynamically in an arbitrary manner. These flexibility in designing the network formation helps to design a network anywhere and anytime. This makes MANET's suitable for many different applications ranging from military to commercial. And also, these are very much suitable for disaster management, rescue operations and defense applications.

The major concern in MANET is the conservation of energy due to the limited lifetime of mobile devices. All the mobile nodes depend on the battery power supply. The main objective of the work here is to make the 802.11 PSM suitable for MANET multihop with Dynamic Source Routing (DSR) protocol. The overhearing problem is due to the integration of DSR protocol with 802.11 PSM (Hu and Hou, 2005; $\mathrm{Li}$ and $\mathrm{Li}, 2005)$.
Another problem with DSR arises due to broadcast flood of control packets ( $\mathrm{Li}$ and $\mathrm{Li}, 2005$ ). So to overcome this, a conditional overhearing and rebroadcast mechanism based on mobility parameter of the nodes in a network is designed and is based on the concept given in RandomCast (Lim et al., 2009). MANET routing protocolperformance mainly depends on the network conditions. The parameters that have an impact on the routing protocol are mobility of the nodes, volume of traffic, busy/idle time of the shared medium and the received signal strength. The ondemand routing protocols DSR (Johnson and Maltz, 1996; Johnson, 2003) and AODV (Perkins and Royer, 1999), before sending a packet to the destination, discovers a route. Route maintenance is invoked when node detects link failure. In order to avoid route discovery for each packet, on-demand routing protocols utilizes cache routes previously learnt. The performance of routing protocol, such as packet endto-end delay, throughput and routing overhead, is

Corresponding Author: Bhanumathi, V., Department of Electronics and Communication Engineering, Anna University of Technology Coimbatore, Coimbatore, India 
significantly influenced by node mobility (Jayakumar and Gopinath, 2008).

DSR gathers the route information through overhearing. Overhearing improves the routing efficiency in DSR by eaves dropping other communications to gather route information but it spends a significant amount of energy. In the Random Cast mechanism (Lim et al., 2009), a node may decide not to overhear i.e., a unicast message and not to forward i.e., a broadcast message when it receives an advertisement during an ATIM window, thereby reducing the energy cost without affecting the network performance. In addition to the energy consumption, overhearing brings in several undesirable features. It could aggravate the stale route problem, the main cause of which is node mobility. So, overhearing and stale route reduction based on mobility of the nodes is considered for the proposed design. But AODV takes a conservative approach to gather route information (Perkins and Royer, 1999). It does not allow overhearing and eliminates existing route information using timeout. This necessitates more RREQ messages which in turn results in more control overheads in routing.

The performance of on-demand routing protocols, therefore, heavily depends on efficient implementation of route cache. This involves cache structure, cache capacity and cache timeout policy. The time varying topology of ad-hoc network, which results from node movement, makes cache entry stale over a period of time. Therefore, a cache timeout policy (Shukla, 2007) is required to predict route cache lifetime and to eliminate stale route cache entries, which are timed out. Deriving proper cache timeout policy thus plays an important role for ensuring cache freshness.

Many techniques have been proposed for route cache (Lou and Yuguang, 2002) organization and its effect on the performance of on-demand routing protocols. But the concentration of cache timeout policy is very less. It is used in route cache implementation to prevent stale route from being used. So, a cross layer design technique is proposed in this study for reducing the unintended overhearing of neighboring nodes and for reducing the stale route. The first problem is solved based on the mobility of nodes. The second problem is solved with the help of Received Signal Strength (RSS) information.

Mobility status can be predicted based on any one of the factors such as, node degree, link duration and number of link breakages. Link breakages can be determined based on the RSS value provided the interference is assumed to be negligible. The prediction of the link breakage based on RSS may not be correct if the interference is high. The idea is tried with a cross layer framework comprising of physical, MAC and network layers. The Received Signal Strength (RSS) value is derived from the physical layer and is shared among the other two layers. The RSS value is used for finding the minimum transmission power for energy saving.

\section{MATERIALS AND METHODS}

DSR protocol-an overview: The DSR (Johnson, 2003) is an efficient reactive routing protocol designed specifically for use in multi-hop mobile ad-hoc networks. Network nodes cooperate to forward packets for each other to allow communication over multiple "hops" between nodes. Network topology is changing quite often, since the number or sequence of intermediate nodes needed to reach any destination may change at any time. DSR do not use periodic 'hello' messages. The DSR protocol is composed of two mechanisms that work together to allow the route discovery and maintenance of source routes in the ad-hoc network.

DSR always operate in promiscuous listening. So, the node can periodically check its route cache for the needed routes. If the route to the particular destination is not available in the route cache, then DSR initiates the route discovery process. Route discovery is one in which a node $\mathrm{S}$ wishing to send a packet to a destination node D obtains a source route to D. Route Discovery is used only when S attempts to send a packet to $\mathrm{D}$ and does not already know a route to $\mathrm{D}$. Route Maintenance is one in which node $S$ is able to detect, while using a source route to $\mathrm{D}$, if the network topology has changed such that it can no longer use its route to $\mathrm{D}$ because a link along the route no longer works. When it indicates a source route is broken, $\mathrm{S}$ can attempt to use any other route it happens to know to D, or it can invoke Route Discovery.

In DSR, Route Discovery and Route Maintenance each operate entirely "on demand". That is why, it is an on-demand routing protocol. DSR requires no periodic packets of any kind at any layer within the network. When a node has a data packet to send but does not know the routing path to the destination, it initiates the route discovery procedure by broadcasting a control packet, called Route Request (RREQ). When an RREQ reaches the destination, it prepares another control packet, called Route Reply (RREP) and replies back to the source with the complete route information. Upon receiving an RREP, the source saves the route information in its local memory, called route cache, for later uses.

The wireless link break due to the node mobility and an upstream node propagates a RERR packet to 
remove stale route information from route caches of the nodes. RERR information is not propagated "fast and wide". Route caches often contain stale route information for an extensive period of time. Overhearing improves the network performance by allowing nodes to collect more route information. Now, overhearing could make the situation even worse. This is because that DSR generates more RREP packets for a route discovery to offer alternative routes in addition to the principal one. While the primary route is checked for its validity during the communication between the source and the destination, alternative routes may remain in route cache unchecked even after they become stale. This applies also for all their neighbors because they learned and kept them by means of unconditional overhearing.

IEEE 802.11 PSM: IEEE 802.11 wireless LANs have become very popular MAC and PHY layer technologies in ad-hoc networking research. A power management mode is mainly to reduce the energy costs of the idle state, but it exhibits poor latency performance in multi hop infrastructure less environments. The power management of DCF is mainly done through the Adhoc Traffic Indication Mode (ATIM) which is transmitted in a special interval directly after the beacon. Many methods to improve the performance of multi hop ad-hoc networks under power management have been proposed. Monarch version (Dorsey and Siewiorek, 2004) of the network simulator is used for the 802.11 power management in ad hoc networks. By using the DCF mechanism, enabling the features of PSM is difficult, because it does not contain an access point. Nodes in the PS mode are expected to synchronize among themselves in a distributed way (Huang and Lai, 2002; Tseng et al., 2003). In ad-hoc environment, source should inform by giving an advertisement through ATIM during the packet Advertisement period (ATIM window). Source will transmit the data during the data transmission period. Here in PSM, every node operates in power save mode and it is not switching between Active Mode (AM) and Power Save mode (PS). It is operated so to minimize the energy consumption and this in turn increases the life time of the network.

ODPM: ODPM (Zheng and Kravets, 2003) is one of the most competitive energy efficient schemes developed for multi-hop networks. In ODPM, a node switches between the AM and PS mode based on communication events and event induced time-out values. ODPM is designed in such a way that every node switches between the AM and PS mode frequently, which leads to non-negligible overhead. One more criteria here is that each node must know the power management mode of its neighbours. The following values are suggested in the study. A node remains in AM for $5 \mathrm{sec}$ until it receives an RREP. It remains in AM for $2 \mathrm{sec}$ if it receives a data packet or it is a source or a destination node. So, it requires an additional energy cost or an extended packet delay. Also, its performance greatly depends on time-out values and it has to be improved based on the routing protocol and traffic conditions. If data traffic is infrequent, the node either stays in AM for all the consecutive intervals without receiving any further data packets or switches to a low-power sleep state. Packet delay is not improved in this method, but it consumes more energy than 802.11 PSM.

RandomCast: RandomCast (Lim et al., 2009) is a new communication mechanism, in which a sender can specify the desired level of overhearing, making a prudent balance between energy and routing. RandomCast is highly energy-efficient compared to conventional 802.11 as well as 802.11 PSM-based schemes, in terms of total energy consumption, energy goodput and energy balance. The ATIM window size and beacon interval used here is 0.05 and $0.25 \mathrm{sec}$. All nodes consistently operate in power save mode and the level of overhearing and rebroadcast level are determined based on the number of neighbors. The overhearing probability is inversely proportional to the number of neighbors. This study compares the performance of RandomCast with 802.11, 802.11 PSM and ODPM, Rcast in terms of PDR, energy consumption, energy goodput and energy balance through simulation. This study identifies four factors that must be considered for the overhearing/rebroadcast decision. These are sender ID, number of neighbors, mobility and remaining battery energy. RandomCast is based only on the second factor (number of neighbors).

Our proposed work is based on the RandomCast and the design is carried out based on the mobility factor. The proposed cross layer design is discussed in the following section.

Cross-layer design: A cross layer design is designed by covering the bottom three layers such as physical, MAC and network layer. The diagrammatic representation of the design is shown in Fig 1. The physical layer transfers the received signal strength value to the top layers. The RSS value is captured for determining the cache expiry of all the individual links, so as to conserve the energy, at the same time life time 
of the battery will be improved. Henceforth, it improves the overall lifetime of the network. Mobility can be determined based on the connectivity changes with the neighbors. Connectivity change is found out using the value of RSS of the selected links.

Mobility is defined as the average change in distance over time between all nodes (in $\mathrm{m} / \mathrm{sec}$ ). When mobility of the nodes in a network is high, link errors frequently occur and this results in high stale route information in the route cache in DSR. Therefore, number of nodes that overhear is kept at a moderate level based on the RSS value. This ensures the stability of the selected route for a certain period of time. From this, it is clear that RSS determines the link quality. If RSS value is more, link quality will be good, otherwise link will likely to be broken soon.

A transmitting node is able to specify the level of overhearing based on mobility which in turn depends on RSS value. The RSS value of these links such as link $(i, j)$ are calculated based on the formula given in Eq. 1:

$\operatorname{RSS}=\mathrm{P}_{\mathrm{t}}(\lambda / 4 \pi \mathrm{d}){ }^{\mathrm{n}} \mathrm{G}_{\mathrm{t}} \mathrm{G}_{\mathrm{r}}$

Where:

$$
\begin{aligned}
& \lambda=\text { Wavelength } \\
& \mathrm{P}_{\mathrm{t}} \quad=\text { Transmit power } \\
& \mathrm{G}_{\mathrm{t}} \text { and } \mathrm{G}_{\mathrm{r}}=\text { Represents the unity gain of the }
\end{aligned}
$$

The Eq. 1 is modified based on the assumption that the interference is negligible and it is given in Eq. 2:

$\operatorname{RSS}=\mathrm{P}_{\mathrm{t}} / \mathrm{d}^{2}$

The RSS threshold $\left(\mathrm{RSS}_{\text {thres }}\right)$ value for finding the overhearing and rebroadcast probability $\left(\mathrm{P}_{\mathrm{OR}}\right)$ is calculated based on the Eq. 3 as follows:

$\mathrm{RSS}_{\text {thres }}=\mathrm{P}_{\mathrm{t}} / 2$

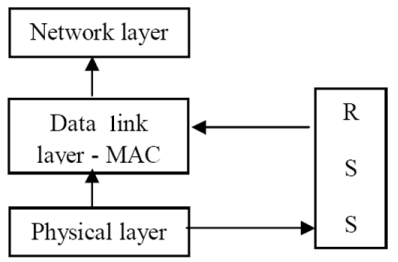

Fig. 1: Cross layer framework
Normally, as the distance increases, the received signal strength starts decreasing. This increases the chances of link breakage between two nodes.

Mobility model is chosen as Random Way Point (RWP) model (Bettstetter et al., 2004). In this model, a mobile node moves on a finite continuous plane from its current position to a new location by randomly choosing its destination coordinates, its speed of movement and the amount of time that it will pause when it reaches the destination. On reaching the destination, the node pauses for some time distributed according to some random variable and the process repeats itself. Once the pause time expires, the node chooses a new destination, speed and pause time.

The Mobility Parameter (MP) of the link is found out using the following formula given in Eq. 4:

$\mathrm{MP}=\mathrm{RSS} / \mathrm{P}_{\mathrm{t}}$

Based on the mobility parameter value, overhearing and Rebroadcast Probability $\left(\mathrm{P}_{\mathrm{OR}}\right)$ is calculated based on the Eq. 5:

$\mathrm{P}_{\mathrm{OR}}=\mathrm{RSS}_{\text {thres }} / \mathrm{RSS}$

In the proposed architecture, $\mathrm{P}_{\mathrm{OR}}$ value is kept lower compared to MP for maintaining the conditional overhearing level of unicast packets as moderate. RREP here is a unicast packet. It is desired that the nodes which are present in the RREP packet should have a higher RSS value, then only it can withstand for some time. It improves the lifetime of the route. MP value is kept higher than the $\mathrm{P}_{\mathrm{OR}}$. This ensures a higher value of RSS. And it is approximately greater than $70 \mathrm{dBm}$ by assuming $100 \mathrm{dBm}$ as the transmit power. The condition of MP greater than or equal to $\mathrm{P}_{\mathrm{OR}}$ results in reduced overhead and thus improve the network performance. And also number of times, the route discovery initiated will also get reduced.

$\mathrm{P}_{\mathrm{OR}}$ value is kept higher compared to MP for the conditional overhearing of Broadcast packet i.e., RREQ. The reason for keeping this value as aggressive is that the RSS value is nominal. So, the nodes are not allowed to overhear, but it is made to rebroadcast the RREQ it received for the conditional overhearing. The possible values of MP and $\mathrm{P}_{\mathrm{OR}}$ is tabulated in Table 1 for various RSS values by keeping the transmit Power $\left(\mathrm{P}_{\mathrm{t}}\right)$ as constant value of $100 \mathrm{dBm}$.

Table: $1 \mathrm{MP}$ and $\mathrm{P}_{\mathrm{OR}}$ values for different RSS values

\begin{tabular}{llc}
\hline RSS $(\mathrm{dBm})$ & MP & $\mathrm{P}_{\mathrm{OR}}$ \\
\hline 50 & 0.5 & 1.00 \\
60 & 0.6 & 0.83 \\
70 & 0.7 & 0.71 \\
80 & 0.8 & 0.62 \\
90 & 0.9 & 0.55 \\
100 & 1.0 & 0.50 \\
\hline
\end{tabular}




\begin{tabular}{|c|c|c|c|c|c|c|c|}
\hline $\begin{array}{l}\text { FC } \\
\text { (2) }\end{array}$ & $\begin{array}{l}\text { DI } \\
\text { (2) }\end{array}$ & $\begin{array}{l}\text { DA } \\
(6)\end{array}$ & $\begin{array}{l}\text { SA } \\
\text { (6) }\end{array}$ & $\begin{array}{l}\text { Bssid } \\
\text { (2) }\end{array}$ & $\begin{array}{l}\mathrm{SC} \\
\text { (2) }\end{array}$ & $\begin{array}{l}\text { Frame } \\
\text { body } \\
\text { (2) }\end{array}$ & $\begin{array}{l}\text { FCS } \\
\text { (4) }\end{array}$ \\
\hline \multicolumn{8}{|c|}{ FC-Frame Control } \\
\hline \multicolumn{6}{|c|}{ DA-Destination Address } & \multicolumn{2}{|c|}{ SA-Source Address } \\
\hline \multicolumn{6}{|c|}{ BSSID-IBSS addresses } & \multicolumn{2}{|c|}{$\mathrm{SC}$-sequence contro } \\
\hline
\end{tabular}

Fig 2: ATIM Frame Format (Length in Bytes)

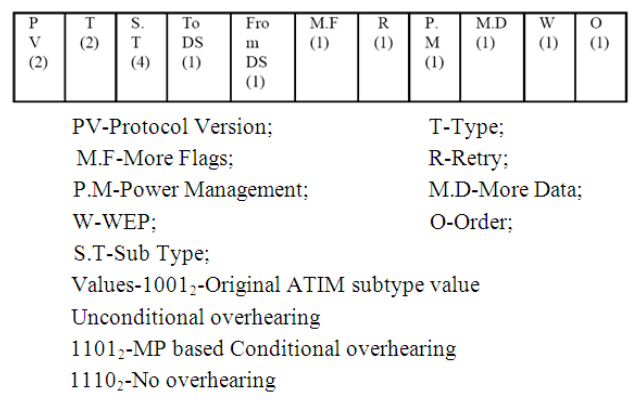

Fig. 3: Frame control field (length in bits) of ATIM frame format

The packets considered here are broadcast and unicast. A device in PS mode periodically wakes up during the packet advertisement period, called Ad-hoc Traffic Indication Message (ATIM) window, to see if it has any data to receive. ATIM frame format is shown in Fig. 2. ATIM frame format of IEEE 802.11 is modified a little to specify the level of overhearing for the nodes. Suppose, the packet is of broadcast type, when a node sends an ATIM frame, all the neighbors will receive the packet and rebroadcast it. The overhearing considered here is of three types. They are no overhearing; Mobility Parameter (MP) based conditional overhearing and unconditional overhearing. The frame control field of ATIM frame format is shown in Fig. 3. The numbers within the brackets of the fields of the frame control field represents the length in bits.

As per the IEEE 802.11 standard, ATIM frame is a management frame of type 002 and its subtype is 10012 which is in general denotes unconditional overhearing. The proposed MP based conditional overhearing takes two unused subtypes such as 11012 and 11102, to specify MP based conditional and no overhearing.

The Subtype field which is included in the frame control header specifies the function for the specified frame type. For example, if the frame is a type management frame, the subtype field indicates the type of management frame (e.g., a beacon frame, authenticate request, or disassociate notice). And here it is used for defining the overhearing level.

A node in an ad-hoc network learns routing information by overhearing or forwarding packets to other nodes and keeps the learned routes in the route caches. The proposed cross layer design is discussed in the following paragraphs.

When a node receives an ATIM frame for a unicast packet, it goes to its wake up state at the beginning of a beacon interval. ATIM frame contains the Destination Address (DA) and subtype value. Based on the address and subtype value, the node concludes whether or not to receive or overhear the advertised packet in the following data transmission period. The node has to wakeup or to sleep is basedon the following conditions:

- The receiving node is the anticipated destination

- The receiving node is not the destination and the source node likes unconditional overhearing

- The receiving node is not the destination, but based on mobility level, the MP based conditional overhearing is applied

DSR utilizes RREQ, RREP and RERR as control packets for route discovery and maintenance. For data transfer, it uses DATA packet. The MP based overhearing mechanism is proposed as follows for the DSR packets:

- MP based conditional overhearing for RREP packets-It is not necessary that RREP packets should be overheard unconditionally by all the nodes in the network. But DSR creates RREP from all the nodes

- MP based conditional overhearing for data packetsData packets contain the entire route information and this can be controlled, so that all the nodes cannot overhear

- Unconditional overhearing for RERR packetsRERR is used to inform the link breakage, so it can be overheard by all the nodes. By unconditionally broadcasting the RERR packets, the network performance can be improved by removing the stale route in route cache and thus by eliminating the broken links

- Conditional overhearing for RREQ packetsNumber of nodes that can overhear RREQ packets, can be controlled based on the mobility level

The algorithm is designed in such a way that employs MP based conditional, unconditional and no overhearing. Broadcast packets are RREQ and Address Resolution Protocol (ARP). RREQ is given conditional overhearing based on rebroadcast probability (POR). ARP is usually unconditionally broadcasted in order to find out the corresponding IP address for a given MAC address. Here MAC address is taken as NA.

The steps for transmitting an ATIM frame in the MP based conditional overhearing are as follows: 
- First RREQ packet will be broadcasted to all the nodes in the network

- The overhearing level will be set in the frame type field of ATIM for the unicast data packets like DATA and RREP

- Nodes in the network may overhear the RREP and stores the route information in route caches based on the subtype

- If there is any link break, RERR is propagated to the source node by an upstream node so that the link can be deleted from route cache and RERR is treated here as unconditional

- Route cache is updated based on the value of RSS received from the physical layer

The following Fig. 4 shows the procedure for the implementation of MP based conditional overhearing mechanism. The key design criteria for the algorithms RSS value based on which a node can select the overhearing level. The algorithm is designed based on the factor mobility. RSS is considered here as one of the metric for deciding the mobility of the nodes in the network. If the RSS value is low, then it is assumed that the nodes are far away and it may soon break.

The above algorithm does not consider the other factors like node ID and residual energy of the nodes. Node ID will help in finding the redundant request very soon, so that delay in finding the routes is avoided. If residual energy is considered in communication, the life time of the network can be improved further.

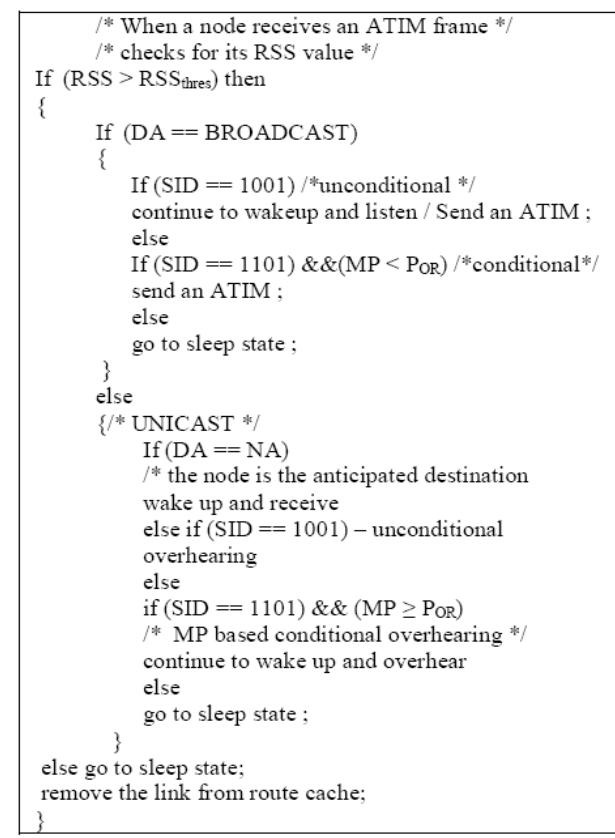

Fig. 4: MP based conditional overhearing

\section{RESULTS}

For the evaluation of the proposed methodology, network simulator 2 VINT Project, 2009 under Linux platform is chosen as the simulation tool. The ad-hoc network topology with 50 nodes in an area of $1000 \times 1000 \mathrm{~m}^{2}$ was considered. The transmission range of the network setup is $250 \mathrm{~m}$. The simulator set up has been done based on the idea given in (Dorsey and Siewiorek, 2004). The channel data rate is chosen as 2 Mbps. The data traffic is Constant Bit Rate (CBR) traffic. The packet rate injected in the network varies from 1-5 packets/sec with a packet size of 256 byte data packet every second. Random waypoint mobility model is considered for the simulation with a maximum node speed of $5 \mathrm{~m} \mathrm{sec}^{-1}$ and a pause time of $0-600 \mathrm{sec}$. With this mobility model, a node travels at the selected mobility speed toward a randomly selected destination in the network. After the node arrives at the location, it pauses for a predetermined period of time and then travels toward another randomly selected location. Total Simulation time is $600 \mathrm{sec}$ and each simulation scenario is repeated 10 times to obtain steady state value.

The proposed cross layer design is compared with 802.11 without PSM, 802.11 with PSM and RandomCast. The ATIM window size and beacon interval selected for the design is 0.05 and 0.25 . The ATIM window size is selected as $1 / 5$ times the beacon interval, to obtain a reasonable throughput. Figure 5 presents Packet Delivery Ratio (PDR) in \% for the packets injected per second. Figure 6 shows a comparative analysis for the Energy consumption by varying the packet rate. Figure 7 represents the energy consumption of all the 50 nodes in a network by injecting the packet at a rate of 2 packets/sec and keeping the node pause time as 0 sec. Figure 8 represents the energy consumption of all the 50 nodes in a network for the packet injection rate of 2 packets/sec. Figure 9 shows an analysis of energy consumption for various pause time.



Fig. 5: Packet delivery ratio analysis 


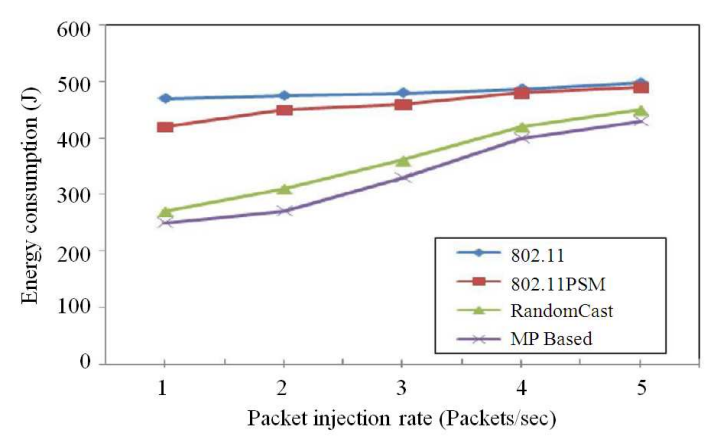

Fig. 6: Energy consumption Vs packet injection rate

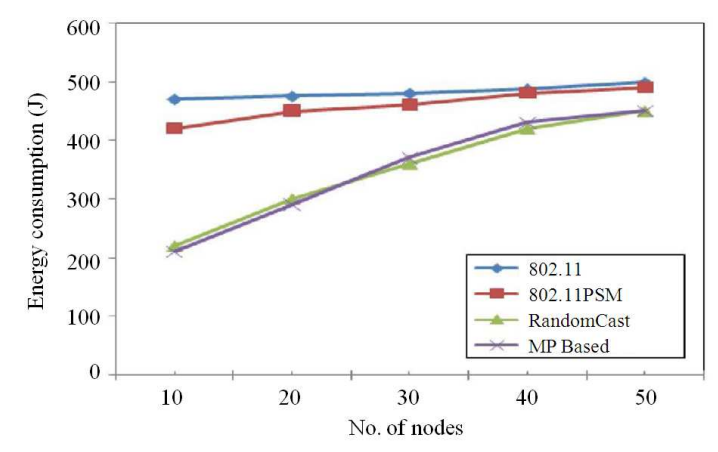

Fig. 7: Energy consumption Vs No. of nodes at pause time $=0 \mathrm{sec}$

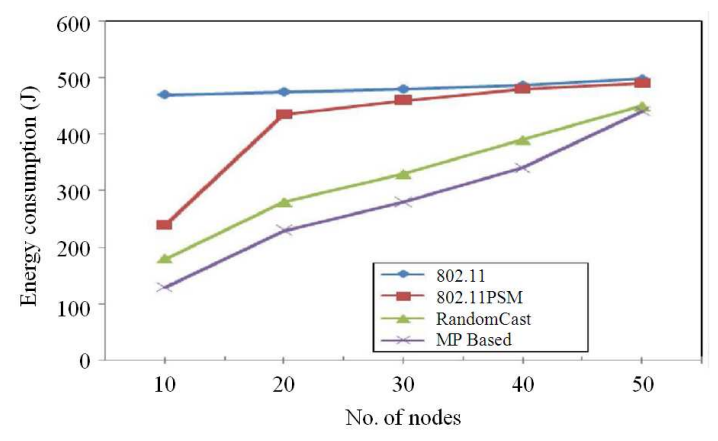

Fig. 8: Energy consumption Vs No. of nodes at pause time $=600 \mathrm{sec}$

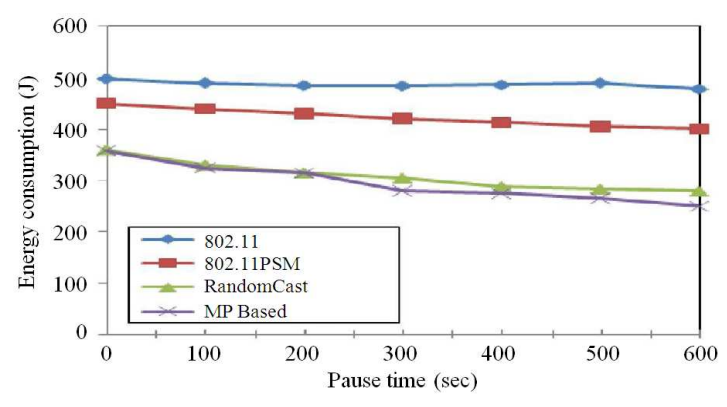

Fig. 9: Energy consumption Vs pause time

\section{DISCUSSION}

As seen from Fig. 5, packet delivery ratio is almost above $90 \%$ up to the packet injection rate of 3 packets/sec. Above this packet rate, only 802.11 show a high packet delivery ratio because all active nodes participate in transmission. The decrease in PDR value for 802.11 PSM, RandomCast and MP based are due to the power save mode of operation. MP based conditional overhearing shows an improvement of 3.2\% over RandomCast algorithm. This is due to the elimination of the links below the RSSthres value.

It is clearly seen from Fig. 6, that the energy consumption of MP based method is very much lesser compared to 802.11 and 802.11 PSM. MP based achieves approximately 30 joules of less energy consumption compared to RandomCast. For the pause time of 0 seconds, the rebroadcast probability is kept higher. As seen from the Fig. 7, energy consumption is almost as same as that of RandomCast.

The graph 8 is drawn by keeping the node pause time as maximum of simulation time $600 \mathrm{sec}$. There is no mobility in the network. Because of the lower overhearing probability, it results in less route discovery and link breakage. The proposed design achieves energy consumption of almost 50 joules lesser compared to RandomCast.

From the graph of Fig. 9, it is seen that as the pause time increases, there is a reduction in average energy consumption value compared to other schemes. Under low mobility, beyond the pause time of 300s, MP outperforms RandomCast and results in an average additional energy saving of $20 \%$. This is due to the reason, that the design takes into consideration only the stable paths with high RSS value.

\section{CONCLUSION}

It is seen from the simulation analysis that energy is utilized effectively in MANET by the cross layer framework implementation. It is designed in such a way that the sender can specify the desired level of overhearing based on the RSS of the link. Whether to select the route or not is based on the RSS value taken from the physical layer which prevents stale routes from being used. This link elimination before transmitting the data reduces the frequency of failures and route discoveries and in turn decreases the overhead also. PDR of MP based design achieves 3.2\% improvement compared to the existing systems. It achieves a significant power saving, if the pause time crosses $300 \mathrm{sec}$. For the packet injection rate of 2 packets/sec, under no mobility condition, MP method 
achieves 50 joules lesser energy consumption compared to RandomCast. Under high mobility condition, it works more or like the RandomCast. The main drawback in the design is the delay, because a packet is advertised before an actual delivery and the announcement can be made only one hop at a time in each beacon interval $(0.25 \mathrm{sec})$. As a future work, a path maintenance mechanism can be implemented to avoid the path breakages. We like to analyze by considering other mobility metrics for the evaluation of the mobility parameter for defining the overhearing level.

\section{REFERENCES}

Bettstetter, C., H. Hartenstein and X. Perez-Costa, 2004. Stochastic properties of the random waypoint mobility model. Wireless Netw., 10: 555-567. DOI: 10.1023/B:WINE.0000036458.88990.e5

Dorsey, J. and D.P. Siewiorek, 2004. 802.11 Power Management Extensions to Monarch NS. 1st Edn., School of Computer Science, Carnegie Mellon University, Pittsburgh, pp: 43.

$\mathrm{Hu}, \mathrm{C}$. and J.C. Hou, 2005. A Link-indexed statistical traffic prediction approach to improving IEEE 802.11 PSM. J. Ad Hoc Netw., 3: 529-545. DOI: 10.1016/j.adhoc.2004.08.003

Huang, L. and T.H. Lai, 2002. On the scalability of IEEE 802.11 ad hoc networks. Proceedings of the 3rd ACM International Symposium on Mobile Ad Hoc Networking and Computing, Jun. 09-11, ACM, New York, pp: 173-182. DOI: $10.1145 / 513800.513822$

Jayakumar, G. and G. Gopinath, 2008. Performance comparison of two on-demand routing protocols for ad-hoc networks based on random way point mobility model. Am. J. Applied Sci., 5: 659-664. DOI: 10.3844/ajassp.2008.659.664

Johnson, D.B. and D.A. Maltz, 1996. Dynamic source routing in ad hoc wireless networks. Kluwer Academic Publishers.
Johnson, D.B., 2003. The dynamic source routing protocol for mobile ad hoc networks (DSR). National Institute of Informatics.

Li, Z. and B. Li, 2005. Probabilistic power management for wireless ad hoc networks. Mobile Netw. Appli., 10: 771-782. DOI: 10.1007/s11036-005-3370-y

Lim, S., C. Yu and C.R. Das, 2009. RandomCast: An energy-efficient communication scheme for mobile ad hoc networks. IEEE Trans. Mobile Comput., 8: 1039-1051. DOI: 10.1109/TMC.2008.178

Lou, W. and F. Yuguang, 2002. The effects of cache organizations on the performance of on-demand routing protocols in ad hoc networks. Proc. MILCOM., $\quad 1$ : 254-259. DOI: 10.1109/MILCOM.2002.1180449

Perkins, C.E. and E.M. Royer, 1999. Ad-Hoc ondemand distance vector routing. Proceedings of the 2nd IEEE Workshop on Mobile Computing Systems and Applications, Feb. 25-26, IEEE Xplore Press, New Orleans, LA , USA., pp: 90100. DOI: 10.1109/MCSA.1999.749281

Shukla, A., 2007. Ensuring cache freshness in ondemand routing protocols for mobile ad hoc network: A cross-layer framework. Proceedings of the 4th IEEE Consumer Communications and Networking Conference, Jan, 11-13, IEEE Xplore Press, Las Vegas, NV, USA., pp: 264-268. DOI: 10.1109/CCNC.2007.59

Tseng, Y.C., C.S. Hsu and T.Y. Hsieh, 2003. Powersaving protocols for IEEE 802.11-based multi-hop ad hoc networks. Comput. Netw., 43: 317-337. DOI: 10.1016/S1389-1286(03)00284-6

Zheng, R. and R. Kravets, 2003. On-demand power management for ad hoc networks. Proceedings of the IEEE Societies 22nd Annual Joint Conference of the IEEE Computer and Communications, Mar. 30-Apr. 3, IEEE Xplore Press, pp: 481-491. DOI: 10.1109/INFCOM.2003.1208699 